\title{
Eletrodos modificados com hidróxido de níquel para determinação de uréia
}

\section{Nickel hydroxide modified electrodes for urea determination}

\author{
Ricardo Curilazo후 Marcelo Rodrigues da Silva²; Luiz Henrique Dall’Antonia ${ }^{3}$
}

\section{Resumo}

Os filmes de hidróxido de níquel foram preparados por eletrodeposição a partir de uma solução 0,05 mol L ${ }^{-1}$ de $\mathrm{Ni}\left(\mathrm{NO}_{3}\right)_{2}$ sobre eletrodos de níquel e ITO (óxido de estanho dopado com índio) sobre filme plástico tipo PET, aplicando-se uma corrente de $-0,1 \mathrm{~mA} \mathrm{~cm}^{-2}$ durante diferentes intervalos de tempo entre 1800 e $7200 \mathrm{~s}$. O comportamento eletroquímico do eletrodo de hidróxido de níquel foi investigado

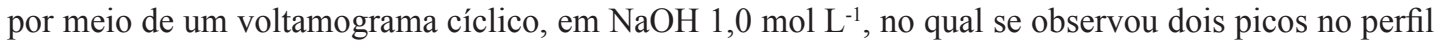
de corrente em função do potencial em 0,410 e $0,280 \mathrm{~V}$, correspondendo ao par redox $\mathrm{Ni}(\mathrm{II}) / \mathrm{Ni}(\mathrm{III})$. Um sensor para uréia apresentando uma resposta satisfatória pode ser obtido quando após o depósito do filme de $\mathrm{Ni}(\mathrm{OH})_{2}$ sobre o eletrodo de níquel, o mesmo for imerso em uma solução de $\mathrm{NaOH} \mathrm{1,0} \mathrm{mol} \mathrm{L-1}$ e aplicando-se um potencial de $+0,435 \mathrm{~V}$, onde ocorre o máximo de corrente anódica no voltamograma cíclico. Analisando, os dados, observa-se que para um determinado intervalo de concentração do analito entre 5 a $50 \mathrm{~m} \mathrm{~mol} \mathrm{~L}^{-1}$, o comportamento apresenta-se linear e a sensibilidade encontrada foi de 20,3 $\mathrm{mA} \mathrm{cm}{ }^{-2}\left(\mathrm{~mol} \mathrm{~L}^{-1}\right)^{-1}$. Ele se apresenta reprodutível e ratifica a utilização de eletrodos de hidróxido de níquel para a determinação de uréia.

Palavras-chave: Uréia. Sensor. Hidróxido de níquel.

\begin{abstract}
Nickel hydroxide films were prepared by electrodeposition from a solution $\mathrm{Ni}(\mathrm{NO} 3) 20,05 \mathrm{~mol} \mathrm{~L}^{-1}$ on ITO electrodes (Tin oxide doped with Indium) on PET-like plastic film, applying a current of $-0,1 \mathrm{~A} \mathrm{~cm}^{-2}$ during different time intervals between 1800 and $7200 \mathrm{~s}$. The electrochemical behavior of the nickel hydroxide electrode was investigated through a cyclic voltammogram, in $\mathrm{NaOH} 1,0 \mathrm{~mol} \mathrm{~L}^{-1}$, where it was observed two peaks in the profile in 0,410 and $0,280 \mathrm{~V}$, corresponding to redox couple $\mathrm{Ni}(\mathrm{II}) / \mathrm{Ni}(\mathrm{III})$. A sensor for urea presenting a satisfactory answer can be obtained when, after the deposit of the film of $\mathrm{Ni}(\mathrm{OH}) 2$ on the electrode of nickel, it is immersed in a solution of $\mathrm{NaOH} 1,0 \mathrm{~mol} \mathrm{~L}^{-1}$ and applying a potential of $+0,435 \mathrm{~V}$, where the maximum of the anodic current occurs in the cyclic voltammogram. Analyzing the results it can be observed that, for a range of analite concentration between 5 to $50 \mathrm{~m} \mathrm{~mol} \mathrm{~L}^{-1}$, the behavior is linear and the sensibility found was of $20,3 \mathrm{~mA} \mathrm{~cm}^{-2}\left(\mathrm{~mol} \mathrm{~L}^{-1}\right)^{-1}$, presenting reproducibility confirming the nickel hydroxide electrodes utilization for the determination of urea.
\end{abstract}

Key words: Urea. Sensor. Nickel hydroxide.

\footnotetext{
${ }^{1}$ Bacharel em Química - Laboratório de Eletroquímica e Materiais/Depto. Química/CCE/UEL

${ }^{2}$ Licenciado em Química - Laboratório de Eletroquímica e Materiais/Depto. Química/CCE/UEL

${ }^{3}$ Professor Adjunto - Laboratório de Eletroquímica e Materiais/Depto. Química/CCE/UEL - luizh@uel.br
} 


\section{Introdução}

O hidróxido de níquel é usualmente empregado como material ativo para eletrodos positivos de níquel e mecanismos eletrocrômicos, e também pode atuar como promotor catalítico para reações que envolvem a evolução de oxigênio. Dessa forma, ele passa a ter considerável para a ciência e tecnologia.

Muitas propriedades do eletrodo de hidróxido de níquel (o alto poder de densidade, a ótima capacidade cíclica e a alta energia específica) proporcionam-lhe grande viabilidade para uma extensa faixa de aplicação (TESSIER et al., 1999). Essas propriedades justificam-se pela existência de quatro fases no período de vida útil do eletrodo de hidróxido de níquel: $\alpha-\mathbf{N i}(\mathbf{O H})_{2}, \gamma-\mathbf{N i O O H}, \beta-$ $\mathbf{N i}(\mathbf{O H})_{2}$ e $\beta$ - NiOOH, cujas reações eletroquímicas podem ser descritas por (1):

$$
\begin{array}{ccc}
\alpha-\mathrm{Ni}(\mathrm{OH})_{2} & \Leftrightarrow & \gamma-\mathrm{NiOOH} \\
\Uparrow & & \mathbb{1} \\
\beta-\mathrm{Ni}(\mathrm{OH})_{2} & \Leftrightarrow & \beta-\mathrm{NiOOH}
\end{array}
$$

Ao analisar, tais fases polimórficas presentes no período de vida útil do eletrodo de hidróxido de níquel, verifica-se que o ciclo $\alpha \Leftrightarrow \gamma$ possui um par de vantagens sobre o ciclo $\beta \Leftrightarrow \beta$. Primeiramente, são trocados cerca de 1,7 elétrons por átomo de níquel durante o ciclo reversível $\alpha$ $\Leftrightarrow \gamma$, enquanto o ciclo $\beta \Leftrightarrow \beta$ troca somente 1 elétron, em conseqüência do estado de oxidação do átomo de níquel no primeiro ciclo ser mais elevado. A segunda vantagem consiste no fato de que, sob a ação de uma carga prolongada, a fase $\beta$ - NiOOH converte-se para a fase $\gamma-\mathbf{N i O O H}$, e isso provoca uma deformação mecânica, a qual resulta em danos irreversíveis, e afeta significativamente as características do eletrodo. Por outro lado, a transição de fase que ocorre no ciclo reversível $\alpha \Leftrightarrow \gamma$ não provoca tal deformação, e torna-se, dessa forma, um melhor material eletroativo, de que resulta um aumento na eficiência do eletrodo de hidróxido de níquel (Tessier e colaboradores, 1999).

A concentração de uréia no sangue e na urina atua como um indicador de doenças renais no ser humano, tornando tal análise muito freqüente em laboratórios clínicos. Entretanto, as medidas necessárias para a quantificação da uréia utilizam métodos convencionais, como, por exemplo, o uso de indofenol. Em tal método, a uréia presente na amostra é hidrolisada pela enzima uréase, e resulta na produção de bicarbonato e íons amônio (2). Esses íons, na presença de salicilato, hipoclorito e nitoprussiato (reação de Berthelot modificada), produzem o corante azul de indofenol, o qual possui um máximo de absorção em torno de $600 \mathrm{~nm}$ e forma uma cor proporcional à concentração de uréia existente.

$$
\begin{gathered}
\mathrm{NH}_{2} \mathrm{CONH}_{2}(\mathrm{aq})+\mathrm{H}^{+}(\mathrm{aq})+2 \mathrm{H}_{2} \mathrm{O}(\mathrm{aq}) \\
2 \mathrm{NH}_{4}^{+}(\mathrm{aq})+\mathrm{HCO}_{3}^{-}(\mathrm{aq})
\end{gathered}
$$

A partir da reação enzimática acima, a determinação de uréia pode ser feita por meio de sensores convencionais, como eletrodos de $\mathrm{pH}$ e eletrodos seletivos a íons amônio, os quais são utilizados para detectar íons hidrogênio e íons amônio, respectivamente.

$\mathrm{O}$ maior problema dos eletrodos de $\mathrm{pH}$, decorre do fato de a resposta do mesmo depender fortemente do meio amostral. Com efeito, uma mudança no $\mathrm{pH}$ ocasionada pelo curso da reação catalisada pela enzima, pode ser mascarada pelo meio utilizado, e disso resulta numa dinâmica bastante restrita do sensor, além de uma baixa seletividade. Para eletrodos sensíveis a íons $\mathrm{NH}_{4}^{+}$, o problema reside na interferência de íons alcalinos como o $\mathrm{Na}^{+}$e $\mathrm{K}^{+}$, presentes no soro fisiológico e na urina. Assim, evidencia-se a importância do desenvolvimento de novas rotas sistemáticas para a determinação de uréia, utilizando mediadores redox na construção de sensores baseados em métodos tais como 
potenciométrico, condutimétrico, amperométrico e pulso amperométrico que substituam a enzima urease no processo de hidrólise da uréia. Disso resultam métodos analíticos mais fáceis, rápidos e economicamente viáveis (BERCHMANS et al., 1998).

O presente trabalho possui como principal objetivo o desenvolvimento de rotas alternativas para a determinação de uréia, com a utilização de sensores amperométricos modificados pela eletrodeposição de hidróxido de níquel. Essa opção é devida ao fato de os mesmos demonstrarem favorecimento à eletrocatálise de diversas reações, dentre as quais, a hidrólise da uréia.

\section{Procedimento Experimental}

Realizou-se a eletrodeposição dos filmes de hidróxido de níquel, como também a análise da resposta dos eletrodos em face a uréia, e utilizouse, nesse procedimento, um potenciostato MQPG modelo 01(Microquímica) interfaciado a um microcomputador para a aquisição de dados.

Os filmes de hidróxido de níquel foram crescidos por eletrodeposição, a partir de uma solução 0,05 mol L-1 de $\mathrm{Ni}\left(\mathrm{NO}_{3}\right)_{2}$ sobre eletrodos de níquel e ITO (óxido de estanho dopado com índio) sobre filme plástico tipo PET. A decomposição foi realizada aplicação de uma corrente de $-0,1 \mathrm{~mA} \mathrm{~cm}^{-2}$ durante diferentes intervalos de tempo entre 1800 e $7200 \mathrm{~s}$.

A análise do sensor foi feita em $25,0 \mathrm{~mL}$ de uma solução 1,0 mol L-1 de $\mathrm{NaOH}$ (Sasaki e colaboradores, 1998), e nela a resposta de corrente elétrica foi medida a $+0,435 \mathrm{~V}$, sendo este o potencial no qual ocorre o máximo de corrente anódica do respectivo voltamograma cíclico. Utilizou-se um eletrodo de $\mathrm{Ag} / \mathrm{AgCl}$ como eletrodo de referência e uma placa de platina como eletrodo auxiliar. Todas as medidas foram realizadas à temperatura ambiente.

\section{Reagentes e Soluções}

Solução de $\mathrm{Ni}\left(\mathrm{NO}_{3}\right)_{2} 0,05 \mathrm{~mol} \mathrm{~L} L^{-1}$ : Pesou-se aproximadamente $0,727 \mathrm{~g}$ de $\mathrm{Ni}\left(\mathrm{NO}_{3}\right)_{2}$ transferindose em seguida esta quantia para um balão volumétrico de $50 \mathrm{~mL}$, no qual se completou o volume com água deionizada. $\mathrm{O}$ reagente utilizado era procedente da $\mathrm{CAAL}^{\circledR}$ com grau P.A e PM=290,81 $\mathrm{g} \mathrm{mol}^{-1}$.

Solução de $\mathrm{NaOH}$ 1,0 mol $\mathrm{L}^{-1}$ : pesaram-se aproximadamente $10 \mathrm{~g}$ do composto transferindo posteriormente para um balão volumétrico de 250 $\mathrm{mL}$ completando-se o volume com água destilada. $\mathrm{O}$ reagente era procedente da marca Synth ${ }^{\circledR}$ e possuía grau P.A - ACS e PM $=40,00 \mathrm{~g} \mathrm{~mol}^{-1}$.

Solução de uréia $0,5 \mathrm{~mol} \mathrm{~L}^{-1}$ : pesou-se aproximadamente $1,5014 \mathrm{~g}$ de uréia com posterior transferência para um balão de $50 \mathrm{~mL}$ de capacidade, no qual se completou o volume com água deionizada. A uréia utilizada era proveniente da ECIBRA $^{\circledR}$ grau P.A - ACS e PM 60,059 $\mathrm{g} \mathrm{mol}^{-1}$.

\section{Resultados e Discussão}

Os filmes de hidróxido de níquel foram depositados por eletrodeposição catódica a partir de uma solução de $\mathrm{Ni}\left(\mathrm{NO}_{3}\right)_{2}$, 0,05 M. Nesse processo, a redução do nitrato a amônio produz hidroxilas aumentando o pH na superfície do eletrodo. Dessa maneira, os íons metálicos reagem com tais hidroxilas precipitando o hidróxido de níquel conforme a reação (3) (Jayashree e colaboradores, 2001):

$$
\begin{gathered}
\mathrm{NO}_{3}^{-}(\mathrm{aq})+7 \mathrm{H}_{2} \mathrm{O}(\mathrm{aq})+8 \mathrm{e}^{-} \rightarrow \\
\mathrm{NH}_{4}^{+}(\mathrm{aq})+10 \mathrm{OH}^{-}(\mathrm{aq}) \\
\mathrm{Ni}^{2+}(\mathrm{aq})+2 \mathrm{OH}^{-}(\mathrm{aq}) \rightarrow \mathrm{Ni}(\mathrm{OH})_{2}(\mathrm{~s})
\end{gathered}
$$


Investigou-se o comportamento eletroquímico do eletrodo de $\mathrm{Ni}(\mathrm{OH})_{2}$ por meio de um voltamograma cíclico como mostrado na Figura 1. Nesta figura, observam-se dois picos nos perfis de corrente, em função do potencial em 0,410 e $0,280 \mathrm{~V}$, os quais correspondem ao par redox $\mathrm{Ni}$ (II) / Ni(III) (KALU et al., 2001; AYASHREE et al., 2001; PROVASI et al., 2001).

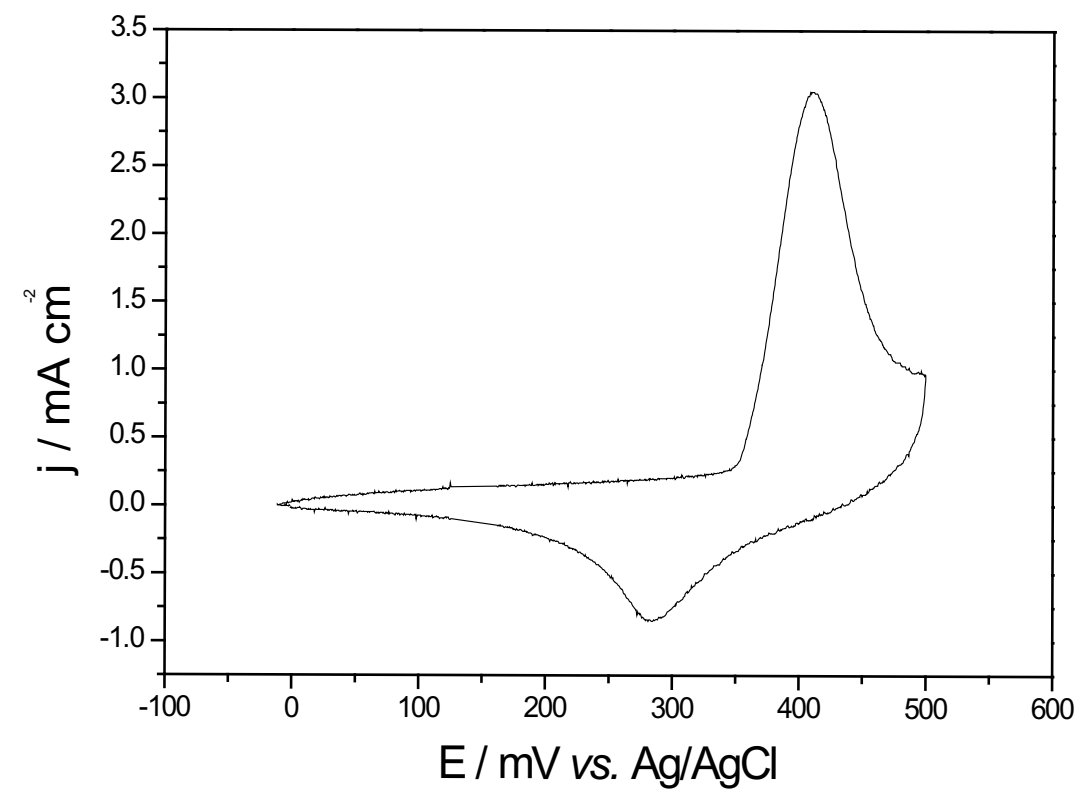

Figura 1. Voltamograma cíclico para eletrodo modificado de $\mathrm{Ni}(\mathrm{OH})_{2}$, depositado por $7200 \mathrm{~s}$. Eletrólito suporte: $\mathrm{NaOH} 1,0 \mathrm{M} \mathrm{e} \mathrm{v}=30 \mathrm{mV} \mathrm{s}^{-1}$.

Obteve-se um sensor para uréia apresentando uma resposta satisfatória, e, após o depósito do filme de $\mathrm{Ni}(\mathrm{OH})_{2}$ sobre eletrodo de níquel durante 7200 segundos, o mesmo foi imerso em uma solução $1,0 \mathrm{~mol} \mathrm{~L}^{-1}$ de $\mathrm{NaOH}$. Aplicou-se um potencial de $+0,435 \mathrm{~V}$, sendo este potencial escolhido de acordo com o voltamograma cíclico, e nessa solução foram adicionadas alíquotas de uréia completando a concentração desejada. Os resultados obtidos são mostrados na Figura 2, na qual se observa que para um determinado intervalo de concentração de uréia entre 5 e $50 \mathrm{~m} \mathrm{~mol} \mathrm{~L}^{-1}$, o comportamento apresenta-se linear (r $=0,998)$, de forma que a sensibilidade encontrada é de aproximadamente $20,3 \mathrm{~mA} \mathrm{~cm}{ }^{-2}\left(\mathrm{~mol} \mathrm{~L}^{-1}\right)^{-1}$, sendo ela correspondente ao coeficiente angular da reta.

Outra maneira utilizada para a investigação do comportamento eletroquímico do eletrodo de hidróxido de níquel frente à uréia foi a aplicação de uma corrente com potencial controlado em $0,435 \mathrm{~V}$, na qual se anotou a respectiva corrente após a mesma estar estabilizada. Tal procedimento foi realizado em $25 \mathrm{~mL}$ de solução $1,0 \mathrm{~mol} \mathrm{~L}^{-1}$ de $\mathrm{NaOH}$ (correspondendo ao branco) e, em seguida, adicionaram-se quantidades de uma solução de uréia. Obteve-se como resultado a curva de calibração, para a determinação amperométrica, mostrada na Figura $3(\mathrm{r}=0,998)$, na qual se observa um aumento na corrente proporcionado pela reação de hidrólise da uréia. De maneira similar, o teste foi repetido com um eletrodo de ITO sem o eletrodepósito de hidróxido de níquel, no qual se observa um aumento de corrente, porém muito abaixo do observado para o eletrodo com depósito de $\mathrm{Ni}(\mathrm{OH})_{2}$. 


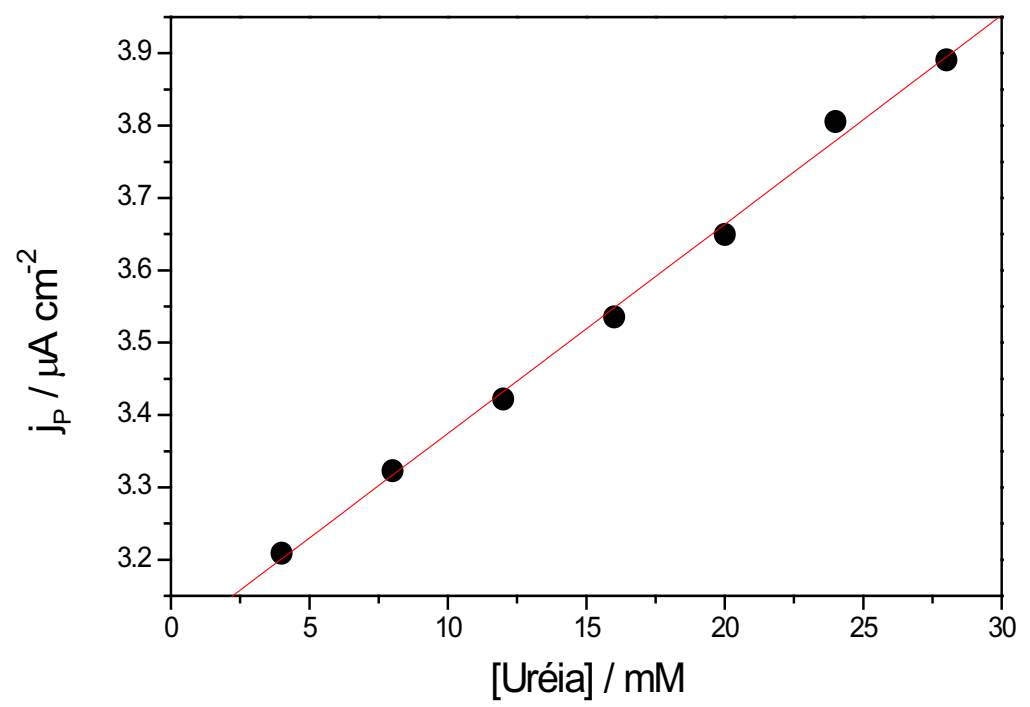

Figura 2. Resposta da densidade de corrente de pico de oxidação $\left(\mathrm{j}_{\mathrm{p}}\right)$ do filme de $\mathrm{Ni}(\mathrm{OH})_{2}$, , depositado por $7200 \mathrm{~s}$. Eletrólito suporte: $\mathrm{NaOH} \mathrm{1,0} \mathrm{M+} \mathrm{uréia.} \mathrm{v}=30 \mathrm{mV} \mathrm{s}^{-1}$. Potencial de pico 0,435 V vs Ag/AgCl.

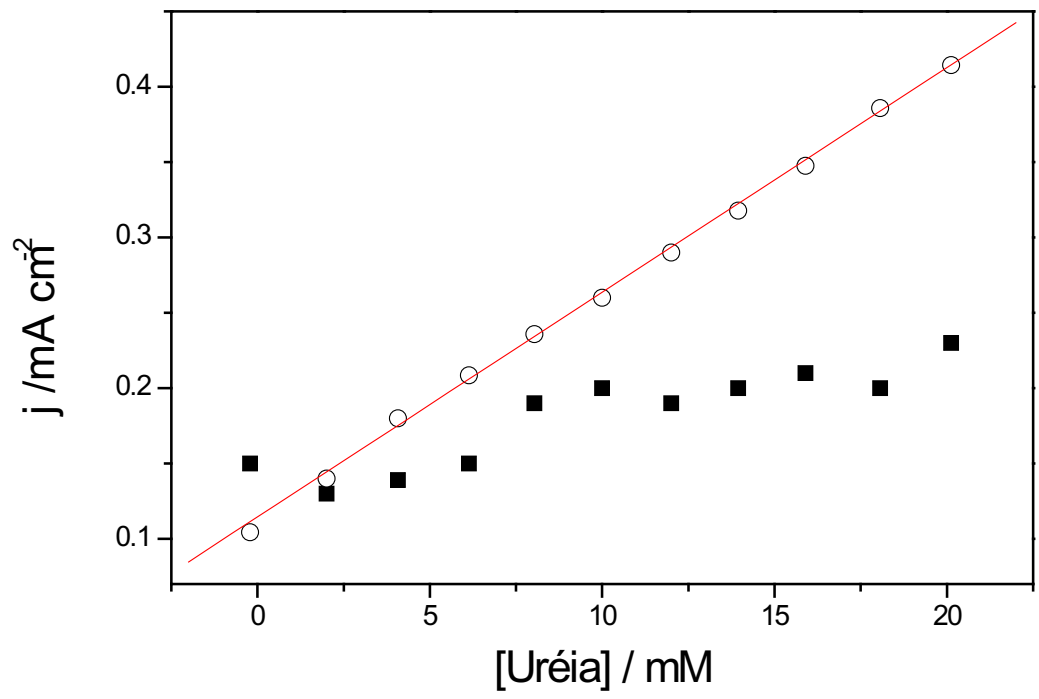

Figura 3. Corrente observada para determinação amperométrica de uréia, sobre eletrodos de de hidróxido de níquel (०) e somente ITO (匹).Potencial aplicado de $+0,435 \mathrm{~V}$ vs Ag/AgCl.

Os produtos da oxidação da uréia provocam um aumento no perfil de corrente, conforme ilustrado na Figura 3, porém os produtos de tal reação ainda são desconhecidos, uma vez que nenhum trabalho presente na literatura descreve algum resultado proeminente, e os experimentos realizados até o presente momento não foram suficientes para elucidar a natureza dos produtos gerados por tal reação. 


\section{Conclusões}

De acordo com os resultados obtidos e relatados no presente trabalho, conclui-se que eletrodos de níquel modificados pela eletrodeposição dehidróxido de níquel apresentam grande reprodutibilidade e eficiência, além de uma ótima reprodutibilidade em face a eletrocatálise da reação de hidrólise da uréia. Isso ratifica a sua utilização como um sensor amperométrico, de maneira que o mesmo se torna uma promissora técnica analítica fácil, rápida e, principalmente, economicamente viável, nos procedimentos para a quantificação da uréia.

\section{Referências}

BERCHMANS, S; GOMATHI, H.; RAO, G. P. Enzymeless approach for the determination of some biologically important species. Sensors and Actuators B, Lausanne, v.50, n.2, p.156-163, 1998.

KALU, E. E.; NWOGA, T. T.; SRINIVASAN, V.; WEIDNER, J. W. Cyclic voltammetric studies of the effects of time and temperature on the capacitance of electrochemically deposited nickel hydroxide. Journal of Power Sources, Lausanne, v.92, n.1/2, p.163-167, 2001.
JAYASHREE, R. S.; VISHNU KAMATH, P. Nickel hydroxide eslectrodeposition from nitrate solutions: mechanistic studies, Journal of Power Sources, Lausanne, v.93, n.1/2, p.273-278, 2001.

PROVASI, K.; GIZ, J. M.; DALL'ANTONIA, L. H.; CÓRDOBA DE TORRESI, S. I.; The effect of Cd, Co and $\mathrm{Zn}$ as additives on nickel hydroxide opto-electrochemical behavior. Journal of Power Sources, Lausanne, v.102, n.1/2, p.224-232, 2001 .

SASAKI, Y.; YAMASHITA, T. Effect of electrolytic conditions on the deposition of nickel hydroxide. Thin Solid Films, Lausanne, v.334, n.1/2, p.117-119, 1998.

TESSIER, C.; HAUMESSER, P. H.; BERNARD, P.; DELMAS, C. The structure of $\mathrm{Ni}(\mathrm{OH})_{2}$ : From the Ideal Material to the Electrochemically Active One, Journal of The Electrochemical Society, Baltimore, v.146, n.6, p.2059-2067, 1999.

ZHU, W. H.; KE, J. J.; YU, H. M.; ZHANG, D. J. A study of the electrochemistry of nickel hydroxide electrodes with various additives. Journal of Power Sources, Lausanne, v.56, n.1, p.75-79, 1995. 